\title{
Low Loss Tapered Polysilicon Core Fibers
}

\author{
F.H. Suhailin, ${ }^{1,2}$ L. Shen, ${ }^{1}$ N. Healy,${ }^{1,3}$ L. Xiao, ${ }^{1}$ M. Jones, ${ }^{4}$ T. Hawkins, ${ }^{4}$ J. Ballato, ${ }^{4}$ U. Gibson, ${ }^{5}$ and \\ A.C. Peacock ${ }^{1, *}$ \\ ${ }^{1}$ Optoelectronics Research Centre, University of Southampton, SO17 1BJ, UK \\ ${ }^{2}$ School of Fundamental Science, Universiti Malaysia Terengganu, 21300 Kuala Terengganu, Malaysia \\ ${ }^{3}$ Emerging Technology and Materials Group, Newcastle University, NE1 7RU, UK \\ ${ }^{4}$ COMSET, School of Materials Science and Engineering, Clemson University, SC 29634, USA \\ ${ }^{5}$ Department of Physics, Norwegian University of Science and Technology, 7491 Trondheim, Norway \\ *Author e-mail address: fhs1m11@ soton.ac.uk
}

\begin{abstract}
We have fabricated small core polysilicon waveguides by tapering bulk, as-drawn silicon optical fibers. The taper process acts to improve the local crystallinity of the core, resulting in a significant reduction in the material loss.

OCIS codes: (060.2280) Fiber design and fabrication; (060.2290) Fiber materials; (060.4370) Nonlinear optics, fibers; (060.6000) Semiconductor Materials.
\end{abstract}

\section{Introduction}

Polysilicon (p-Si) materials are gaining increased interest for the development of low cost and flexible, CMOS compatible waveguides. However, as of to date, the relatively high reported propagation losses for small core waveguides fabricated on-chip have somewhat hindered their application potential, where, for example, there has yet to be any demonstration of nonlinear device operation [1]. Recent work has shown that the main contribution to the losses is the surface roughness, which is typically enhanced by the grain structure [2]. In contrast, p-Si waveguides that are fabricated within the optical fiber platform have been shown to have near atomically smooth surfaces as the polycrystalline material is confined within the pristine silica glass cladding [3], so that the losses are only limited to that of the bulk material. As a result, this platform offers a route to produce high quality, small core $\mathrm{p}-\mathrm{Si}$ waveguides, with the potential for direct integration with standard fiber infrastructures.

In this paper, we propose and demonstrate a novel approach to obtaining low loss p-Si waveguides with core dimensions of only a few microns, or less, by post-processing crystalline silicon core fiber fabricated via a bulk fiber drawing tower technique [4]. As well as reducing the core dimensions, the tapering procedure also acts to improve the local crystallinity of the p-Si material, resulting in a significant reduction of the losses. This combination of small core sizes and low transmission loss has enabled the first characterization of the nonlinear transmission properties of a p-Si waveguide - either fiber or chip-based. The estimated nonlinear parameters are found to be in good agreement for those of single crystal materials, as expected for high quality $\mathrm{p}-\mathrm{Si}$.

\section{Fabrication and Linear Transmission Characterization}

The original p-Si fiber was fabricated via a conventional fiber drawing tower to have an outer diameter of $\sim 130 \mu \mathrm{m}$ and a core diameter of $\sim 10 \mu \mathrm{m}$. Before tapering, the silicon fiber is first sleeved into a thicker capillary to ensure that the mechanical integrity is preserved. The tapering is then performed using a Vytran GPX-3300 machine, during which the core is molten and the silica cladding is softened. This method is remarkably versatile and fibers have been tapered to different ratios, and thus core sizes, as shown in Fig. 1. Here the fibre core diameters are (a) $~ 4.8$ $\mu \mathrm{m}$, (b) $\sim 3.5 \mu \mathrm{m}$ and (c) $\sim 0.94 \mu \mathrm{m}$. In all cases, the diameters change in proportion to the core, and a smooth transition occurs from untapered section to taper waist. Typical lengths are $\sim 20 \mathrm{~mm}$ for the tapered region, with a constant waist of $\sim 10 \mathrm{~mm}$.
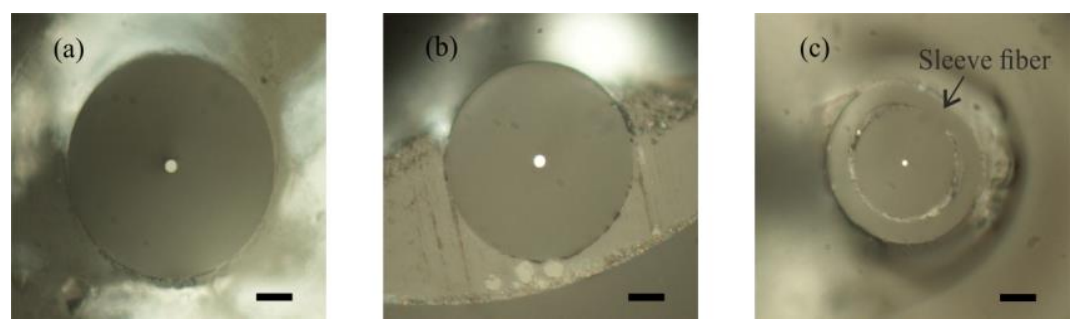

Fig. 1 The cross-section images of the waist of all tapered fibers at the scale bar of $10 \mu \mathrm{m}$. 
To measure the linear transmission losses, the facets of fibers were prepared using a standard polishing technique, following which a CW $1.55 \mu \mathrm{m}$ laser $(\sim 6 \mathrm{~mW})$ was launched into the fundamental mode of the core. The fiber length was cut-back repeatedly until the taper waist was reached, and the transmitted power recorded for each length. The measured losses for the three tapered fibers shown in Fig. 1 are (a) $8.04 \mathrm{~dB} / \mathrm{cm}$, (b) $5.58 \mathrm{~dB} / \mathrm{cm}$ and (c) $3.5 \mathrm{~dB} / \mathrm{cm}$. Comparing these values with that of the untapered fiber, which had a loss of $12.0 \mathrm{~dB} / \mathrm{cm}$, it is clear that the tapering process has helped to improve the crystallinity of the core, with the greatest improvement obtained for the highest taper ratio, i.e., smallest core. To the best of our knowledge, the loss of $3.5 \mathrm{~dB} / \mathrm{cm}$ is the lowest recorded value for a submicron core $\mathrm{p}$-Si waveguide to date [5]. As a result, this tapered fiber presents a unique opportunity to investigate the nonlinear properties of the $\mathrm{p}$-Si material, for the first time.

\section{Nonlinear Transmission Characterizations}

The nonlinear transmission properties were investigated monitoring the propagation of high power $1.54 \mu \mathrm{m}$ laser pulses (720 fs (FHWM) pulse duration and $40 \mathrm{MHz}$ repetition rate) within the small core fiber. The output light was directed either to a power meter or a spectrum analyzer. Fig. 2 (a) shows a plot of the output power as a function of the coupled pump power, which saturates due to the nonlinear two-photon absorption (TPA) and free-carrier absorption (FCA). Figs. 2 (b) and (c) then show the output spectra for two different pump powers, with the high power pump clearly displaying the expected broadening due to the Kerr-induced self-phase modulation. Fitting these results with the standard nonlinear Schrödinger equations for silicon waveguides, as described in [6], reveals the best fit values of the TPA parameter, $\beta_{\mathrm{TPA}} \sim 0.7 \mathrm{~cm} / \mathrm{GW}$, FCA parameter, $\sigma_{\mathrm{f}} \sim 1.45 \times 10^{17} \mathrm{~cm}^{2}$, and the nonlinear refractive index $n_{2} \sim 0.5 \times 10^{-14} \mathrm{~cm}^{2} / \mathrm{W}$. These nonlinear parameters are comparable to those reported for $\mathrm{c}-\mathrm{Si}$ waveguides [7], a good indication of the high crystallinity of the p-Si core.
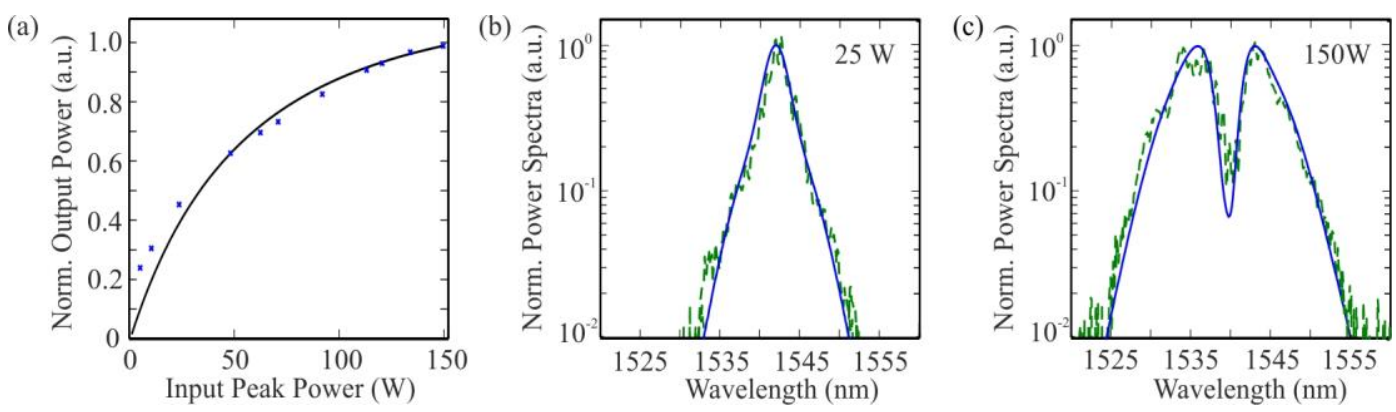

Fig. 2 (a) Nonlinear absorption as a function of coupled input peak power. Spectra evolution at (b) $25 \mathrm{~W}$ and (c) $150 \mathrm{~W}$ coupled peak powers in the small core fiber. Solid curves are for numerical simulation fitting.

In conclusion, we have demonstrated the fabrication of tapered $\mathrm{p}$-Si core fibers via a conventional fiber tapering process. Our modified fiber tapering method opens a route to obtaining low loss, micron-sized core p-Si fibers, which are highly desirable for nonlinear pulse manipulation. The loss value of $\sim 3 \mathrm{~dB} / \mathrm{cm}$ obtained in the smallest tapered core fiber has allowed for the first characterization of the nonlinear transmission properties in a $\mathrm{p}-\mathrm{Si}$ waveguide of any type. Thus, we can expect that with continued improvement to the crystallinity, p-Si waveguides will emerge as desirable components for the development of low cost and flexible nonlinear optical systems.

\section{References}

[1] J. S. Orcutt, S. D. Tang, S. Kramer, K. Mehta, H. Li, V. Stojanović, et al., "Low-loss polysilicon waveguides fabricated in an emulated high-volume electronics process," Optics Express 20, 7243-7254 (2012).

[2] D. Kwong, J. Covey, A. Hosseini, Y. Zhang, X. Xu, and R. T. Chen, "Ultralow-loss polycrystalline silicon waveguides and high uniformity 1x12 MMI fanout for 3D photonic integration," Optics Express 20, 21722-21728 (2012).

[3] N. Healy, L. Lagonigro, J. R. Sparks, S. Boden, P. J. A. Sazio, J. V. Badding, et al., "Polycrystalline silicon optical fibers with atomically smooth surfaces," Optics Letters 36, 2480-2482 (2011).

[4] E. F. Nordstrand, A. N. Dibbs, A. J. Eraker, and U. J. Gibson, "Alkaline oxide interface modifiers for silicon fiber production," Optical Materials Express 3, 651-657 (2013).

[5] N. Healy, S. Mailis, N. M. Bulgakova, P. J. A. Sazio, T. D. Day, J. R. Sparks, et al., "Extreme electronic bandgap modification in lasercrystallized silicon optical fibres," Nat Mater 13, 1122-1127 (2014).

[6] P. Mehta, N. Healy, N. F. Baril, P. J. A. Sazio, J. V. Badding, and A. C. Peacock, "Nonlinear transmission properties of hydrogenated amorphous silicon core optical fibers," Opt. Express 18, 16826-16831 (2010).

[7] A. D. Bristow, N. Rotenberg, and H. M. van Driel, "Two-photon absorption and Kerr coefficients of silicon for 850-2200nm," Appl. Phys. Lett. 90, 191104 (2007). 